\title{
Gut dysbiosis in severe mental illness and chronic fatigue: a novel trans-diagnostic construct? A systematic review and meta-analysis
}

\author{
Jenelle Marcelle Safadi ${ }^{1,2} \cdot$ Alice M. G. Quinton $\mathbb{1}^{2} \cdot$ Belinda R. Lennox ${ }^{2} \cdot$ Philip W. J. Burnet $^{2}$ • \\ Amedeo Minichino $\mathbb{1}^{2}$
}

Received: 12 October 2020 / Revised: 18 December 2020 / Accepted: 13 January 2021 / Published online: 8 February 2021

(c) The Author(s) 2021. This article is published with open access

\begin{abstract}
Reduced gut-microbial diversity ("gut dysbiosis") has been associated with an anhedonic/amotivational syndrome ("sickness behavior") that manifests across severe mental disorders and represent the key clinical feature of chronic fatigue. In this systematic review and meta-analysis, we investigated differences in proxy biomarkers of gut dysbiosis in patients with severe mental illness and chronic fatigue vs. controls and the association of these biomarkers with sickness behavior across diagnostic categories. Following PRISMA guidelines, we searched from inception to April 2020 for all the studies investigating proxy biomarkers of gut dysbiosis in patients with severe mental illness and chronic fatigue. Data were independently extracted by multiple observers, and a random-mixed model was used for the analysis. Heterogeneity was assessed with the $I^{2}$ index. Thirty-three studies were included in the systematic review; nineteen in the meta-analysis $(N=$ 2758 patients and $N=1847$ healthy controls). When compared to controls, patients showed increased levels of zonulin (four studies reporting data on bipolar disorder and depression, $\mathrm{SMD}=0.97 ; 95 \% \mathrm{Cl}=0.10-1.85 ; P=0.03, I^{2}=86.61 \%$ ), lipopolysaccharide (two studies reporting data on chronic fatigue and depression, $\mathrm{SMD}=0.77 ; 95 \% \mathrm{Cl}=0.42-1.12 ; P<$ $0.01 ; I^{2}=0 \%$ ), antibodies against endotoxin (seven studies reporting data on bipolar disorder, depression, schizophrenia, and chronic fatigue, $\mathrm{SMD}=0.99 ; 95 \% \mathrm{CI}=0.27-1.70 ; P<0.01, I^{2}=97.14 \%$ ), sCD14 (six studies reporting data on bipolar disorder, depression, schizophrenia, and chronic fatigue, $\left.\mathrm{SMD}=0.54 ; 95 \% \mathrm{Cl} 0.16-0.81 ; P<0.01, I^{2}=90.68 \%\right)$, LBP (LBP, two studies reporting data on chronic fatigue and depression, $\mathrm{SMD}=0.87 ; 95 \% \mathrm{Cl}=0.25-1.48 ; P<0.01 ; I^{2}=$ $56.80 \%$ ), alpha-1-antitripsin (six studies reporting data on bipolar disorder, depression, and schizophrenia, SMD = 1.23; $95 \% \mathrm{Cl}=0.57-1.88 ; P<0.01, I^{2}: 89.25 \%$ ). Elevated levels of gut dysbiosis markers positively correlated with severity of sickness behavior in patients with severe mental illness and chronic fatigue. Our findings suggest that gut dysbiosis may underlie symptoms of sickness behavior across traditional diagnostic boundaries. Future investigations should validate these findings comparing the performances of the trans-diagnostic vs. categorical approach. This will facilitate treatment breakthrough in an area of unmet clinical need.
\end{abstract}

\section{Introduction}

In the past two decades, the view of severe mental illnesses as brain-centered disorders has been successfully

Supplementary information The online version contains supplementary material available at https://doi.org/10.1038/s41380021-01032-1.

Amedeo Minichino

amedeo.mininichino@psych.ox.ac.uk

1 Cornell University, Ithaca, NY, USA

2 Department of Psychiatry, University of Oxford, Oxford, UK challenged [1]. A growing body of evidence shows that a number of peripheral influences are at play, suggesting that a whole-body perspective might offer greater insight into the understanding of mental health conditions $[2,3]$.

The gut-brain axis, with the relatively recent characterization of the human gut microbiome, is emerging as a key path on the bidirectional communication network between peripheral and central physiological functions [4]. Understanding the relevance of gut microbiome modifications for clinical features of severe mental illness is a research priority, given its modifiable nature and the possibility of unveiling new therapeutic targets in areas of unmet need.

Reduced gut-microbial diversity ("gut dysbiosis") has been associated with a number of detrimental health 
outcomes, including severe mental illness and chronic fatigue [4]. This association appears to occur, at least in part, via a biological pathway that includes the activation of a peripheral pro-inflammatory response [5-7]. Animal models showed that gut dysbiosis triggers a chronic lowgrade pro-inflammatory status in the host by increasing the permeability of the gut barrier ("leaky gut") and by facilitating the translocation of bacterial antigens into the bloodstream ("endotoxemia") (Fig. 1) [5-7]. In both animals and experimental models in otherwise healthy subjects, endotoxemia manifests with a range of flu-like symptoms (fatigue, anhedonia, loss of motivation), which most authors refer to as "sickness behavior" $[8,9]$.

Diagnostic terminology aside [10], symptoms of sickness behavior can be found in all major mental illnesses and beyond, such as in chronic fatigue (Table 1). A recent review [11] nicely summarizes findings from a number of neuroimaging and electrophysiological studies, highlighting a shared central pathophysiology for key sickness behavior symptoms, such as anhedonia and avolition, across different diagnostic categories. Peripheral mechanisms are also shared, with meta-analytic evidence showing increased proinflammatory cytokines levels in schizophrenia and depression [12], and bipolar disorder [13], and an association with severity of sickness behavior symptoms [14]. Similar findings were reported for chronic fatigue [15].

The existence of a shared central and peripheral pathophysiology across major psychiatric illnesses is not surprising, considering the common genetic vulnerability [16]. This common ground may translate in clinical features that manifest across diagnostic boundaries, as hypothesized by a number of clinical and scientific high-profile initiative, such as the Research Domain Criteria [17].

Based on aforementioned considerations, we hypothesize that gut dysbiosis might underlie symptoms of sickness behavior in severe mental illness and beyond, such as in chronic fatigue.

To provide ground for this hypothesis, we conducted a systematic review and meta-analysis aimed at: (i) summarizing the evidence on differences in proxy markers of gut dysbiosis (Fig. 1) in severe mental illnesses (schizophrenia, depression, and bipolar disorder) and chronic fatigue vs. controls; and (ii) investigating the association between these peripheral biomarkers and the severity of sickness behavior symptoms across diagnostic boundaries.

\section{Methods}

This systematic review and meta-analysis was conducted in accordance with the PRISMA guidelines (PRISMA Flowchart in Supplementary). Protocol was registered in PROSPERO. The search for published studies was conducted from inception to April 2020, in Web of Science and PubMed.

Titles and abstracts were imported to Mendeley. We included for full-text analysis: (i) case-control studies reporting data on proxy markers of gut dysbiosis in patients vs. controls; and (ii) studies investigating the association of these biomarkers with symptom severity and treatment response. We included studies investigating patients with a DSM or ICD-codified diagnosis of schizophrenia, depression, and bipolar disorder. We also included studies investigating chronic fatigue syndrome as defined by internationally validated criteria, such as the Fukuda diagnostic criteria. All these conditions manifest with symptoms of sickness behavior, as outlined in Table 1.

The full list of biomarkers was a-priori agreed with an expert (PWJB) after a preliminary search of the literature, and included: tight-junction proteins (zonulin, claudin, etc.); endotoxins (lipopolysaccharide (LPS)); proteins related to the immune response to bacterial antigens (lipopolysaccharide binding protein (LBP) and sCD14); antibodies against bacterial endotoxin, such as anti-Saccharomyces cerevisiae antibodies (Ig-ASCA); proteins related to intestinal inflammation, such as alpha-1-antitrypsin (A-1-AT) and intestinal fatty-acid binding protein (I-FABP).

No restrictions were set in terms of age, duration of illness, and medication status. Reasons for exclusion are documented in the Supplementary. Authors were contacted as needed to determine inclusion. The entire search process was conducted independently by JMS and AMGQ; any disagreements were resolved by AM. Data extraction was independently conducted by JMS and AMGQ.

\section{Outcomes and meta-analyses}

Our primary outcome was to compare differences in circulating levels of proxy markers of gut dysbiosis in patients vs. healthy controls. Our secondary outcomes were: (i) to report data on the relationship between proxy markers of gut dysbiosis and the severity of sickness behavior symptoms (measured across different diagnostic categories, see Table 1); (ii) to report data on the association between modifications of proxy markers of gut dysbiosis and response to treatment. A quantitative synthesis of the differences in proxy markers of gut dysbiosis between patients and controls was provided when data from two or more studies were available for a specific biomarker. Medians, standard errors, and interquartile ranges were transformed to means and SDs, following a validated procedure [18]. When necessary, data were extracted from graphs using a web-based tool (WebPlot Digitizer). All meta-analyses were conducted in R, and the standardized mean difference (SMD) was used as the summary statistic. Heterogeneity between studies was 

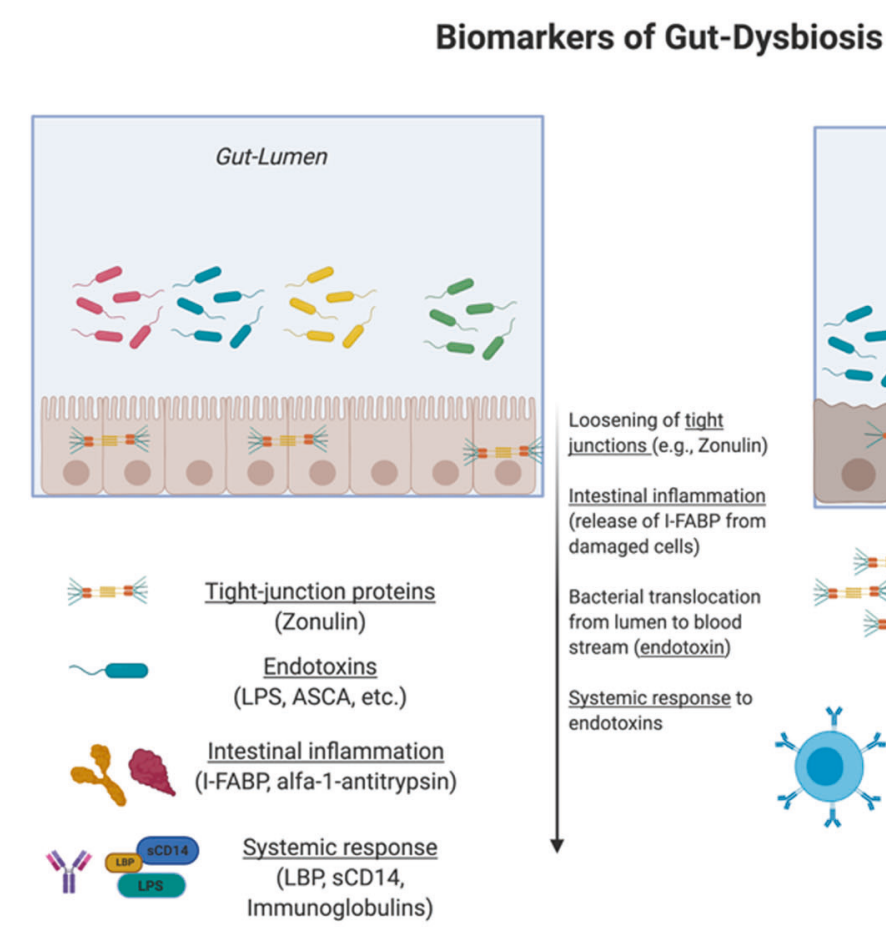

Gut-Dysbiosis

(loss of microbial diversity)
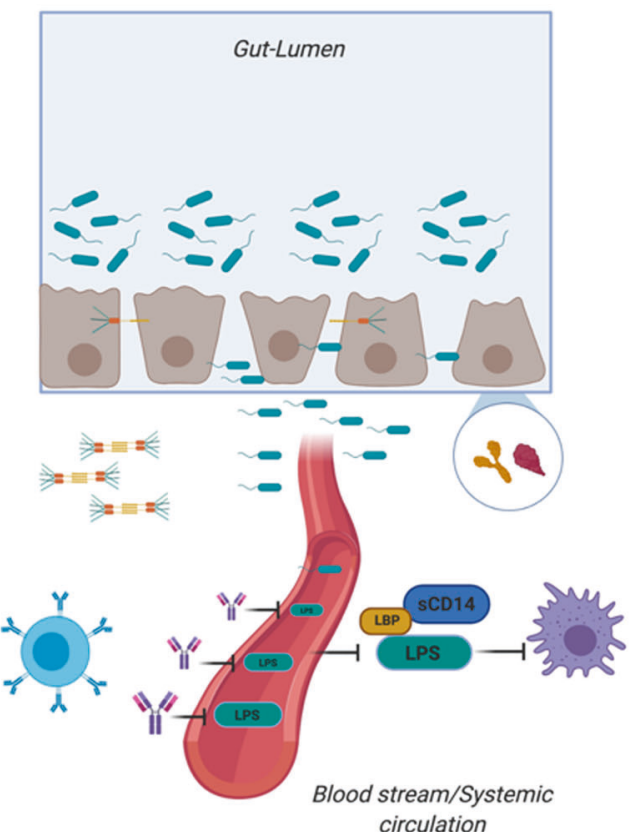

Fig. 1 Proxy biomarkers of gut dysbiosis. Gut dysbiosis (i.e., reduced gut-microbial diversity) has been shown to trigger: a a local inflammatory response (alpha-1-antitrypsin; I-FABP); b loosening of tight-junction proteins (zonulin); $\mathbf{c}$ translocation of bacterial endotoxin from the gut lumen to the bloodstream (LPS, ASCA, etc.); $\mathbf{d}$ activation

of a systemic low-grade inflammation (LBP, sCD14, antibodies against bacterial endotoxin). Experimental models of gut dysbiosis showed a causative link with symptoms of sickness behavior (more details in main text).

Table 1 Symptoms of sickness behavior in schizophrenia (negative symptoms), depression, bipolar disorder (depressive symptoms), and chronic fatigue.

\begin{tabular}{|c|c|c|c|c|}
\hline & $\begin{array}{l}\text { Sickness } \\
\text { behavior }\end{array}$ & $\begin{array}{l}\text { Schizophrenia (negative } \\
\text { symptoms) }\end{array}$ & $\begin{array}{l}\text { Depression and bipolar } \\
\text { disorder (depressive } \\
\text { symptoms) }\end{array}$ & Chronic fatigue syndrome \\
\hline Fatigue & $X$ & $\mathrm{X}$ & $\mathrm{X}$ & $\mathrm{X}$ \\
\hline Malaise & $X$ & & $\mathrm{X}$ & $\mathrm{X}$ \\
\hline Depressed mood & $\mathrm{X}$ & & $\mathrm{X}$ & \\
\hline $\begin{array}{l}\text { Sleep disturbance: insomnia and/ } \\
\text { or hypersomnia }\end{array}$ & $X$ & & $\mathrm{X}$ & $X$ \\
\hline Impaired concentration & $X$ & & $\mathrm{X}$ & $\mathrm{X}$ \\
\hline Curbing of interests & $X$ & $\mathrm{X}$ & $X$ & $X$ \\
\hline Diminished social drive & $X$ & $\mathrm{X}$ & $\mathrm{X}$ & $\mathrm{X}$ \\
\hline Diminished emotional range & & $\mathrm{X}$ & & \\
\hline Anhedonia & $X$ & & $X$ & \\
\hline $\begin{array}{l}\text { Psychomotor retardation or } \\
\text { agitation }\end{array}$ & $\mathrm{X}$ & $X$ & $X$ & \\
\hline Anorexia & $\mathrm{X}$ & & $\mathrm{X}$ & $\mathrm{X}$ \\
\hline Hyperalgesia & $X$ & & & $X$ \\
\hline Pyrexia (fever) & $\mathrm{X}$ & & & $\begin{array}{l}\text { X } \\
\text { Swollen throat and/or } \\
\text { lymph nodes }\end{array}$ \\
\hline Scaled used for assessment & & PANSS negative, SANS & HAMD, BPRS, BDI, CDSS & Fibrofatigue Scale \\
\hline
\end{tabular}

FibroFatigue Scale Fibromyalgia and Chronic Fatigue Syndrome Rating Scale, HAM-D Hamilton Depression Rating Scale, MADRS Montgomery-Åsberg Depression Rating Scale, PANSS-NSS Positive and Negative Syndrome Scale (negative symptom subscale). 
estimated using Higgin's $I^{2}$. Sensitivity analyses were performed by sequentially removing single studies and re-running the analysis. When possible, we explored the effect of medication status on meta-analytic findings by comparing differences in medicated and unmedicated patients. Finally, we reran all the analyses by: (1) removing studies investigating chronic fatigue, to explore if findings were specific for severe mental illness only; (2) removing studies that explicitly included participants in the hypomanic or manic phase of bipolar illness, which do not manifest symptoms of sickness behavior.

\section{Results}

Thirty-three studies met the inclusion criteria [9, 13, 19-49]. Of these, 19 studies $[9,13,19-22,27,29,32-36$, $38,39,41,42,46,49]$ provided data and were included in the meta-analysis $(N=2758$ patients and $N=1847$ healthy controls). It was possible to provide a quantitative synthesis for the following biomarkers: zonulin, LPS, LBP, sCD14, antibodies against bacterial endotoxins, A-1-AT, and I-FABP (Fig. 2).

\section{Differences in proxy markers of gut dysbiosis in patients vs. controls}

Four studies provided data on circulating levels of zonulin in patients $(N=98)$ vs. controls $(N=100)[33,36,38,39]$. Three of these studies were conducted on patients with depression [36, 38, 39] and one on bipolar disorder [33]. Across these four studies, the pooled estimate showed a significant increase in zonulin in patients vs. controls $(\mathrm{SMD}=0.97 ; 95 \% \mathrm{Cl}=0.10-1.85 ; P=0.03)$, with evidence of high heterogeneity $\left(I^{2}=86.61 \%\right)$ (Fig. 2a). Sequentially removing single studies from the analysis did not reduce heterogeneity. However, one study [36], included also patients with anxiety disorders; when this study was removed, the heterogeneity dropped to a moderate level $\left(I^{2}=32.48 \%\right)$, with results being still significant $(\mathrm{SMD}=$ $0.55 ; 95 \% \mathrm{Cl}=-0.12-0.97 ; P=0.011)$.

Only two studies provided data on circulating levels of endotoxins (LPS) in patients $(N=71)$ vs. controls $(N=66)$ $[36,46]$. One of these studies was conducted on patients with depression [36] and the other on chronic fatigue [46]. The pooled estimate showed increased levels of LPS in patients vs. controls (SMD $=0.77 ; 95 \% \mathrm{Cl}=0.42-1.12$; $P<0.01 ; I^{2}=0 \%$ ) (Fig. 2b). A third study [37], not included in the quantitative synthesis, measured LPS in root canal samples of patients with depression and controls. Results from this study were in line with the overall pooled estimate for LPS (Table 2), showing increased levels of LPS in patients vs. controls.
Seven studies [27, 29, 32, 41, 42, 47, 48] provided data on circulating levels of antibodies against bacterial endotoxins in patients $(N=1104)$ vs. controls $(N=603)$. Three of these studies reported data on patients with schizophrenia [27, 31, 47], one on bipolar disorder [32], one on depression [48], one on bipolar disorder and depression [41], and one on chronic fatigue [42].

The pooled estimate showed increased levels of antibodies against bacterial endotoxins in patients vs. controls $(\mathrm{SMD}=0.99 ; 95 \% \mathrm{CI}=0.27-1.70 ; P<0.01)$, with evidence of high heterogeneity $\left(I^{2}=97.14 \%\right.$ ) (Fig. 2c). This high heterogeneity was explained by removing one study [47], with results remaining significant $(\mathrm{SMD}=0.67 ; 95 \%$ Cl $\left.0.44-0.89 ; P<0.01 ; I^{2}=55.67 \%\right)$. Three studies, two on chronic fatigue $[42,45]$ and one on a mixed sample of bipolar disorder and major depression [40], investigated levels of antibodies against bacterial endotoxins in patients vs. controls, but did not provide data. All these three studies reported increased levels of antibodies against bacterial endotoxins in patients vs. controls, in line with our pooled estimate (Table 2).

Six studies $[9,13,22,28,38,46]$ provided data on circulating levels of sCD14 in patients $(N=1234)$ vs. controls $(N=962)$. Two of these studies reported data on patients with bipolar disorder and schizophrenia $[13,22]$, one on bipolar disorder [9], one on schizophrenia [28], one on chronic fatigue [46], and one on depression [38]. The pooled estimate showed increased levels of sCD14 in patients vs. controls $(\mathrm{SMD}=0.54$; 95\% Cl $0.16-0.81 ; P<0.01)$, with evidence of high heterogeneity $\left(I^{2}=90.68 \%\right) \quad$ (Fig. 2d). Sequentially removing single studies from the analysis did not reduce heterogeneity.

Only two studies $[39,46]$ provided data on circulating levels of LBP in patients $(N=71)$ vs. controls $(N=51)$. One study was conducted on patients with depression [39] and the other on chronic fatigue [46]. The pooled estimate showed increased levels of LBP in patients vs. controls $\left(\mathrm{SMD}=0.87 ; 95 \% \mathrm{Cl}=0.25-1.48 ; P<0.01 ; I^{2}=56.80 \%\right)$ (Fig. 2e). Two additional studies that were not included in the quantitative synthesis investigated differences in LBP in patients vs. controls $[22,25]$. The first study reported not significantly higher levels of LBP in bipolar disorder patients compared to controls; however, a quantitative summary was not available [22]. The other study [25] was excluded from the meta-analysis because it was conducted on patients before the onset of schizophrenia (prodrome), showing no differences in LBP circulating levels between patients and controls.

Six studies [19-21, 34, 35, 49] provided data on circulating levels of A-1-AT in patients $(N=342)$ vs. controls $(N=209)$ (Fig. 2f). One of these studies was conducted on patients with bipolar disorder and schizophrenia [20], two 
a. Zonulin

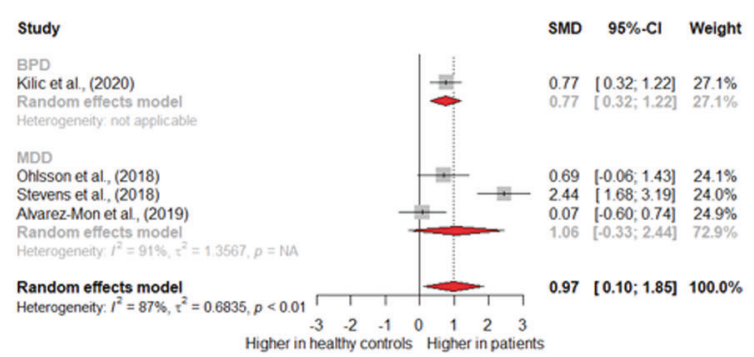

b. LPS

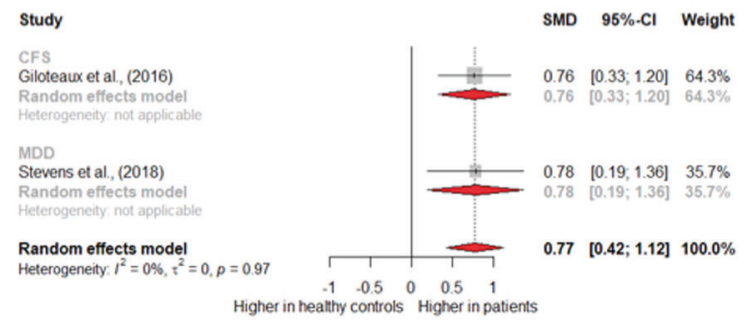

c. Antibodies to endotoxins

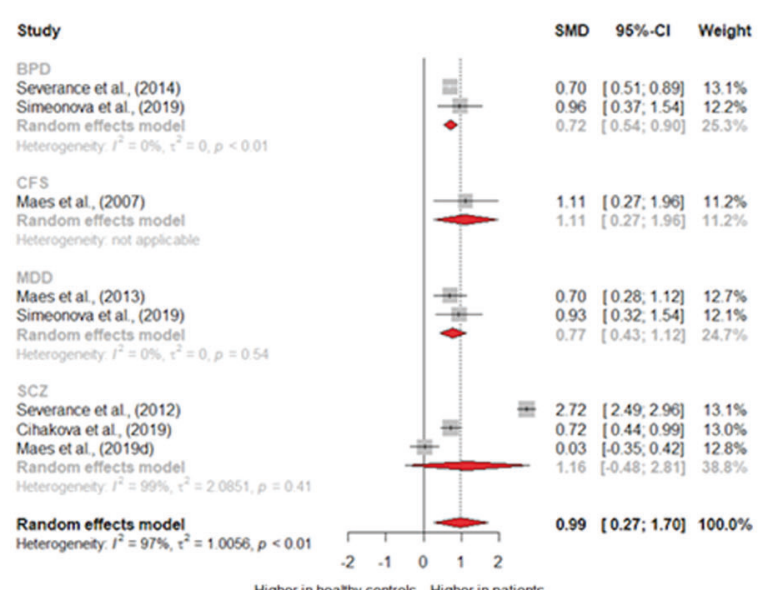

Fig. 2 Forest plots of proxy biomarker of gut dysbiosis in severe mental illness and chronic fatigue. a Levels of circulating zonulin in patients with CFS and MDD vs. controls; $\mathbf{b}$ levels of circulating LPS in patients with CFS and MDD vs. controls; $\mathbf{c}$ levels of circulating antibodies to endotoxins in patient with BPD, CFS, MDD; SCZ vs. controls; d levels of circulating sCD14 in patients with BPD, CFS, MDD, SCZ vs. controls; e Levels of circulating LBP in patients with d. SCD14
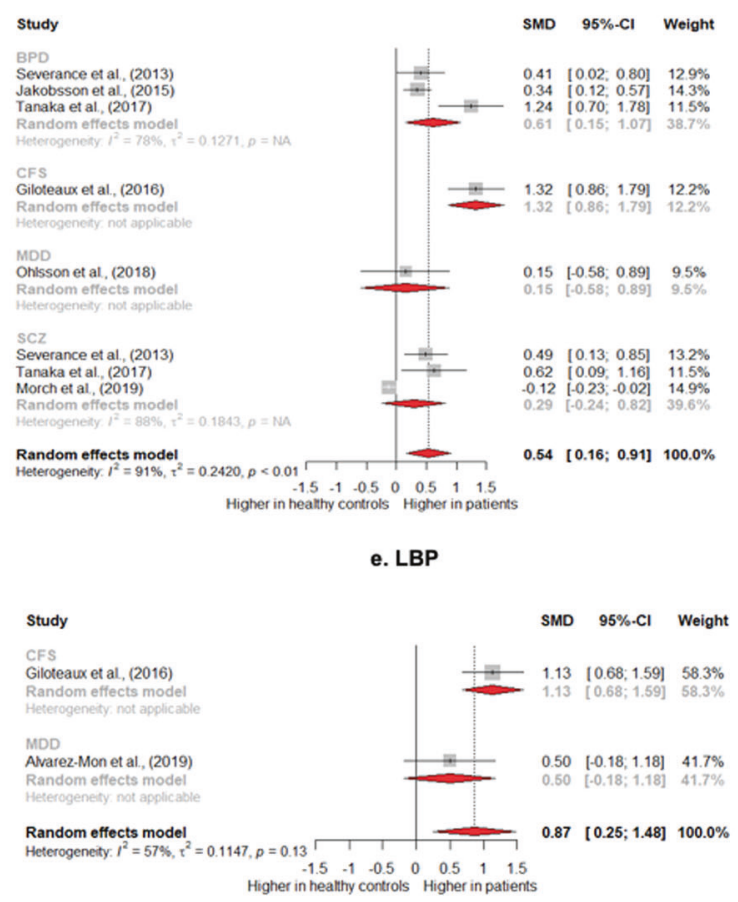

f. A-1-AT

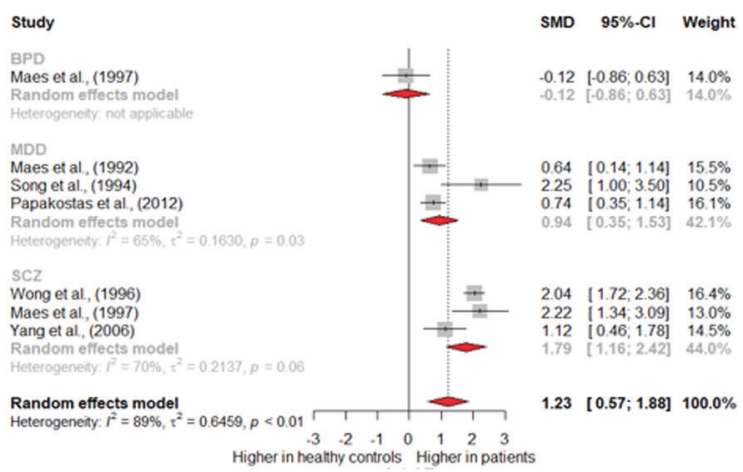

g. I-FABP

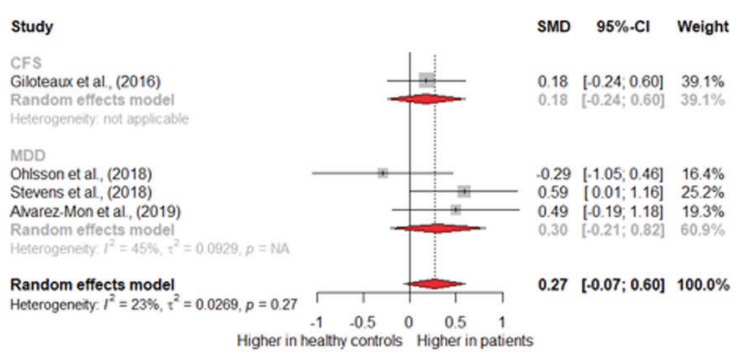

CFS and MDD vs. controls; $\mathbf{f}$ levels of circulating A-1-AT in patients with BPD, MDD, SCZ vs. controls; $g$ levels of circulating I-FABP in patients with CFS and MDD vs. controls. A-1-AT alpha-1-antitrypsin, BPD bipolar disorder, CFS chronic fatigue syndrome, I-FABP intestinal fatty-acid binding protein, LBP lipopolysaccharide binding protein, LPS lipopolysaccharide, MDD major depressive disorder, sCD14 soluble CD14, SCZ schizophrenia. 


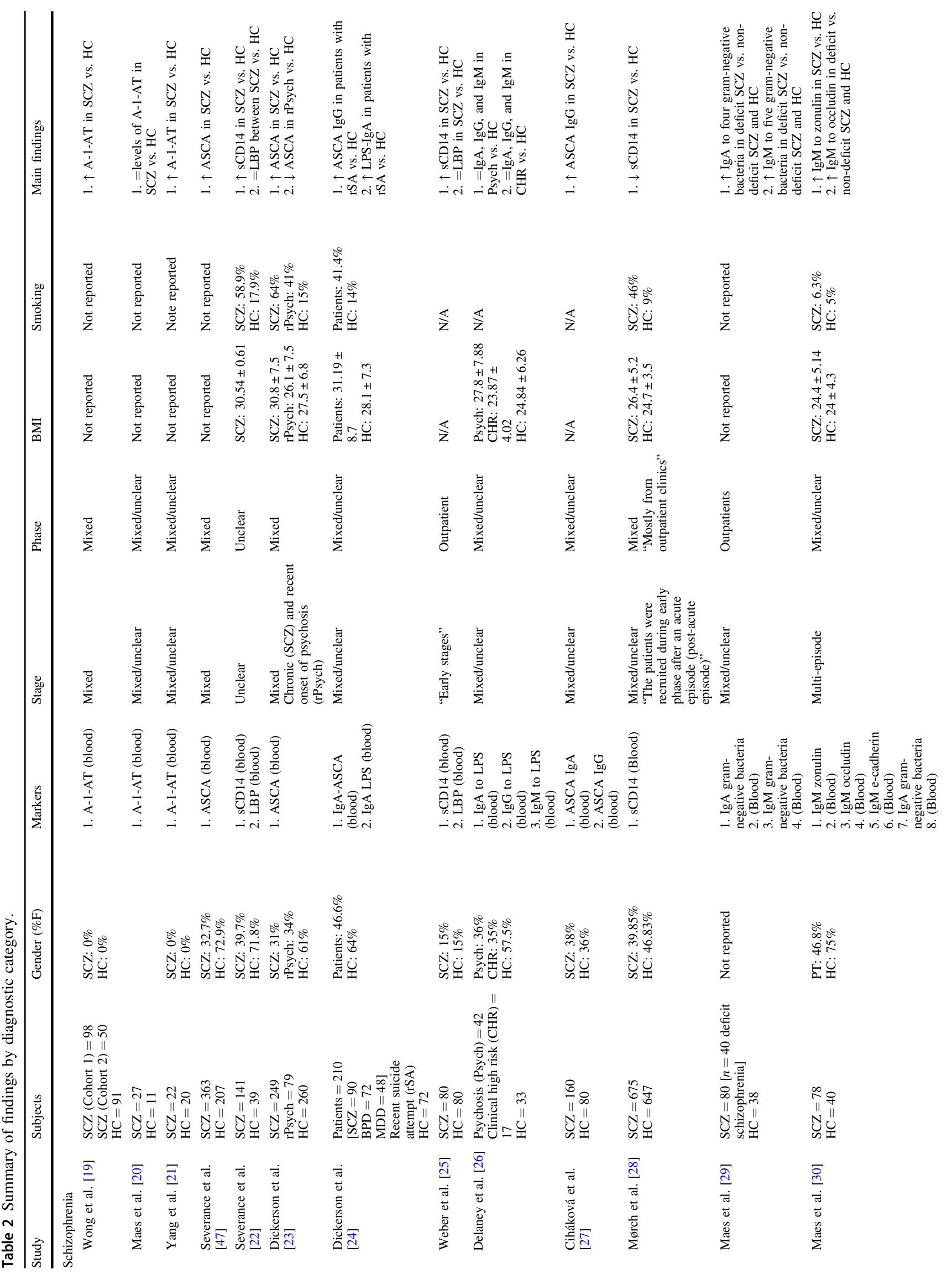




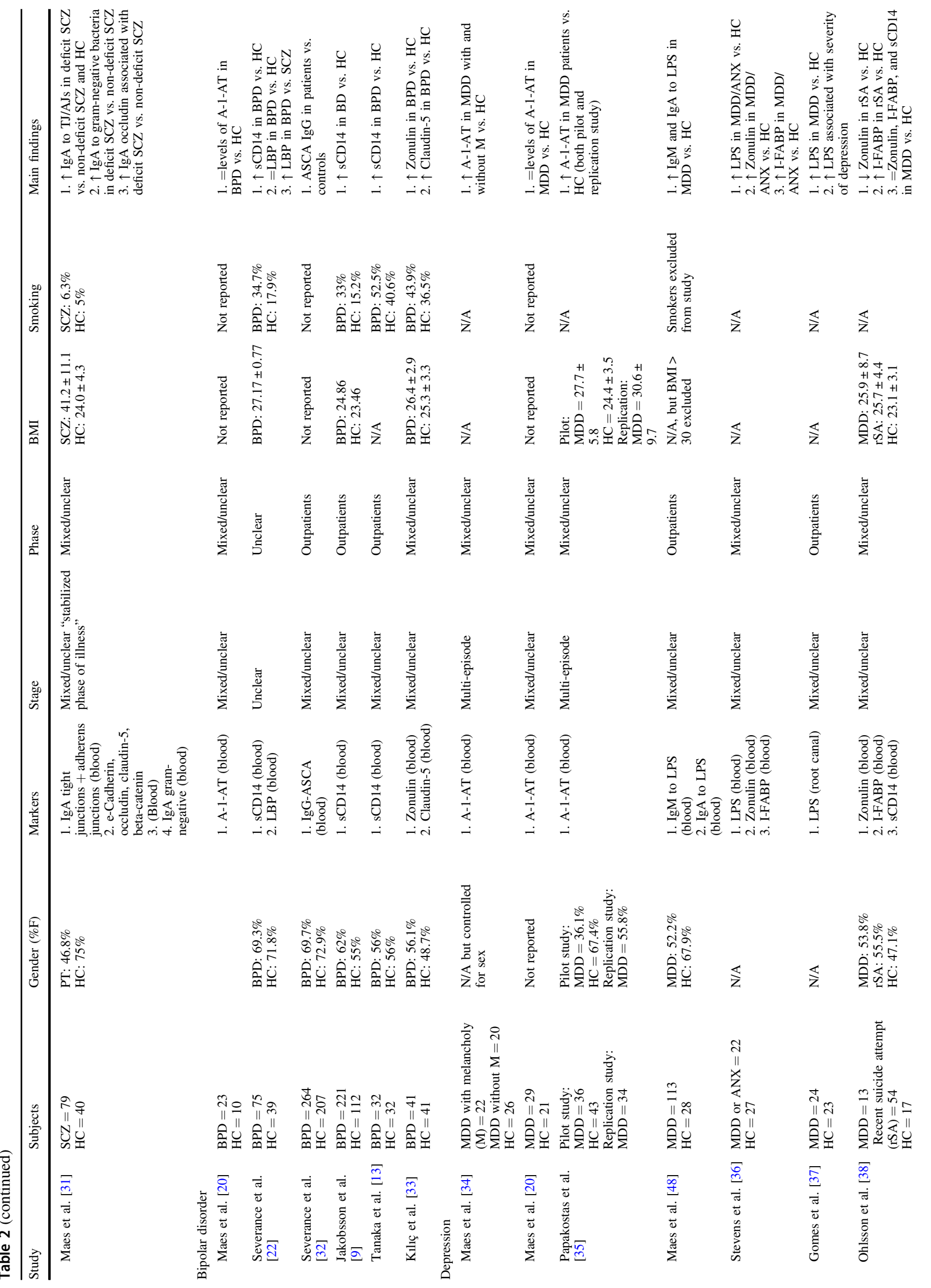




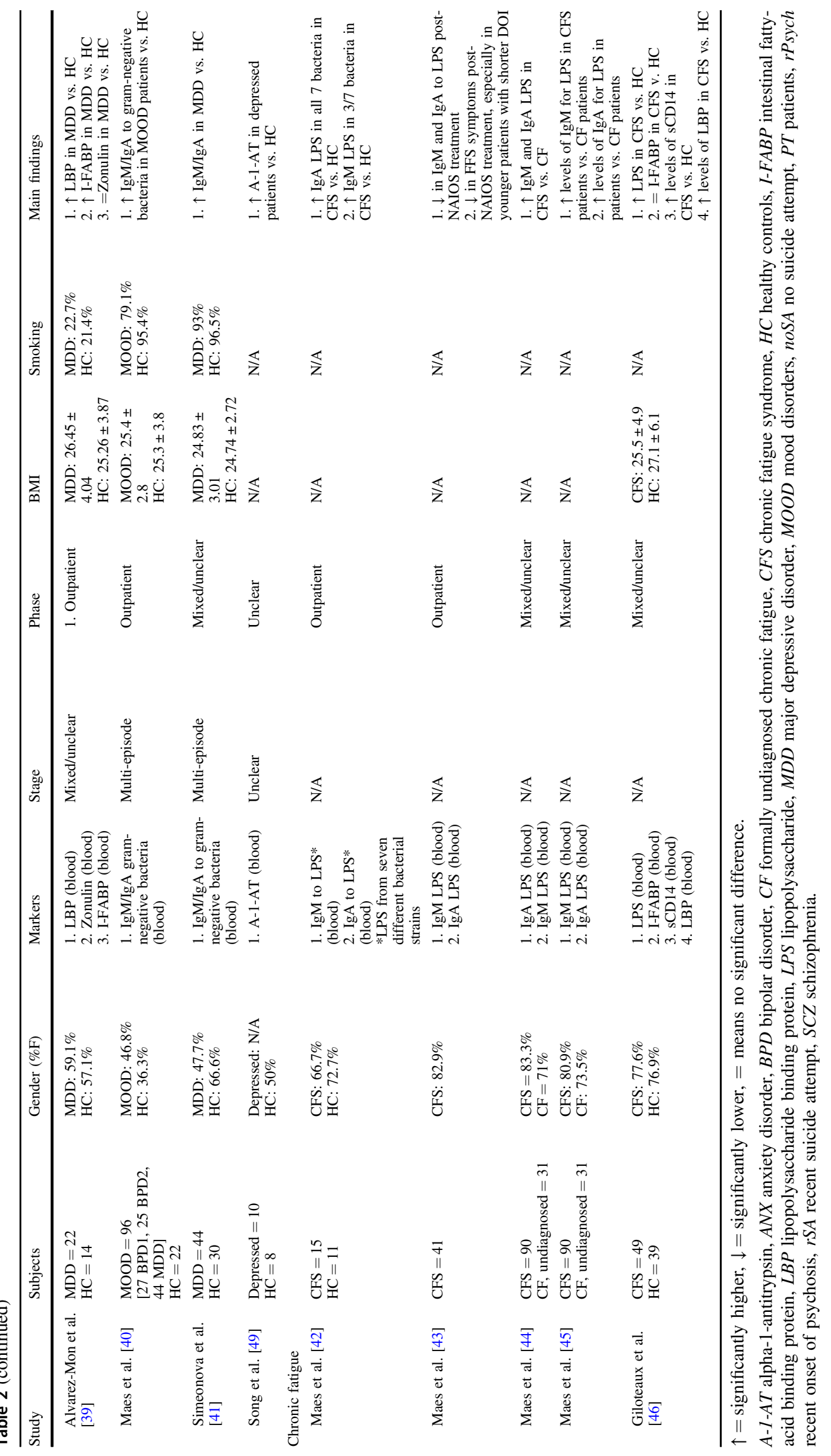


on schizophrenia $[19,21]$, and three on depression [34, 35, 49]. The pooled estimate showed increased levels of A-1-AT in patients vs. controls $(\mathrm{SMD}=1.23 ; 95 \% \mathrm{Cl}=$ $0.57-1.88 ; P<0.01)$, with evidence of high heterogeneity $\left(I^{2}: 89.25 \%\right)$. Sequentially removing single studies from the analysis did not reduce heterogeneity.

Four studies [36, 38, 39, 46] provided data on circulating levels of I-FABP in patients $(N=101)$ vs. controls $(N=$ 92). One of these studies [46] was conducted on patients with chronic fatigue and three on depression [36, 38, 39]. The pooled estimate showed no significant differences in I-FABP levels of patients vs. controls $(\mathrm{SMD}=0.27 ; 95 \%$ $\left.\mathrm{Cl}-0.07-0.60 ; P=0.12 ; I^{2}=23.05 \%\right)$.

In summary, our pooled estimates showed increased circulating levels of: tight-junction proteins (zonulin, four studies reporting data on bipolar disorder and depression); bacterial endotoxins (LPS, two studies reporting data on chronic fatigue and depression); intestinal inflammation markers (A-1-AT, six studies reporting data on bipolar disorder, depression, and schizophrenia); gut-related systemic inflammation markers (LBP, two studies reporting data on chronic fatigue and depression; and sCD14, six studies reporting data on bipolar disorder, depression, schizophrenia, and chronic fatigue); antibodies against endotoxins (seven studies reporting data on bipolar disorder, depression, schizophrenia, and chronic fatigue), in patients compared to controls.

Significance of pooled estimates did not change when studies on chronic fatigue and hypomanic/manic patients with bipolar illness were excluded from the quantitative analyses. Medication status did not influence findings (Supplementary material). As outlined in Table 2, BMI and smoking status were accounted for by in the majority of included studies (Supplementary material).

\section{Association between proxy markers of gut dysbiosis, severity of sickness behavior symptoms, and response to treatment}

The association between circulating levels of proxy markers of gut dysbiosis and severity of sickness behavior symptoms was investigated by 14 studies [13, 23, 28-32, 34, 37, 38, 28-32, 40-42, 44] (Table 3). Of these studies, seven were conducted on patients with schizophrenia [13, 23, 28-31, 40], five on depression [34, 37, 38, 40, 41], four on bipolar disorder [13, 32, 40, 41], and two on chronic fatigue syndrome [42, 44].

Four studies [29-31, 40] showed that greater circulating levels of proxy markers of gut dysbiosis (tight-junction proteins, endotoxins, antibodies against endotoxins) were significantly associated with more severe and persistent negative symptoms (deficit schizophrenia) among patients with schizophrenia. This association was not found in studies including patients with less severe negative symptoms $[13,23,28]$.

Six studies [32, 34, 37, 38, 40, 41] out of seven [13, 32, 34, 37, 38, 40, 41] found that increased circulating levels of proxy markers of gut dysbiosis (A1-AT, I-FABP, endotoxins, antibodies against endotoxins) were significantly associated with more severe symptoms of depression in MDD [34, 37, 38, 40, 41] and bipolar disorder $[32,40]$.

Finally, two studies found that increased circulating levels of proxy markers of gut dysbiosis (antibodies against endotoxins) were significantly associated with more severe symptoms of CFS [42, 44].

Only one study, conducted in patients with chronic fatigue, investigated the relationship between proxy markers of gut dysbiosis and treatment response in patients. This study showed normalization of circulating levels of antibodies against endotoxin after successful treatment [43].

\section{Discussion}

To the best of our knowledge this is the first systematic review and meta-analysis investigating proxy biomarkers of gut dysbiosis in severe mental illness and chronic fatigue.

Gut dysbiosis biomarkers were increased in patients vs. controls and associated with more severe symptoms of sickness behavior across diagnostic categories, independent of medication status.

The pooled estimates showed that patients, when compared to controls, had increased circulating levels of the tight-junction protein zonulin, the endotoxin LPS, the gutrelated systemic inflammatory proteins LBP and sCD14, antibodies against endotoxins, and the acute phase protein A-1-AT.

Zonulin is a tight-junction protein and key regulator of intestinal permeability, with increased circulating levels suggesting a compromised intestinal barrier [6]. Our finding of increased levels of zonulin in patients vs. controls is in line with recent pathophysiological models of psychiatric disorders, where increased permeability of biological barriers, including the blood-brain barrier, is at play [50, 51]. According to these models, the loss of integrity of these protective layers ("leakiness") would result in increased passage to the bloodstream and the brain of "unwanted" material, including "false" neurotransmitters, pro-inflammatory stimuli, and bacterial endotoxins ("endotoxemia"). Accordingly, circulating levels of the bacterial endotoxin LPS were increased in patients compared to controls. The peripheral increase in LPS would also explain findings of increased innate and adaptive response to circulating endotoxins (sCD14/LBP and antibodies) in patients. When the innate immune 
Table 3 Association between proxy markers of gut dysbiosis and severity of sickness behavior in schizophrenia, bipolar disorder, major depression, and chronic fatigue.

\begin{tabular}{|c|c|c|c|c|}
\hline & CFS & Schizophrenia & $\mathrm{BD}$ & MDD \\
\hline Tight-junction proteins & & $++[30,31]$ & & \\
\hline Circulating endotoxin & & & & $+[37]$ \\
\hline sCD14 & & $==[13,28]$ & $=[13]$ & \\
\hline $\begin{array}{l}\text { Antibodies against bacterial } \\
\text { endotoxins }\end{array}$ & $++[42,44]$ & $\begin{array}{l}=[23] \\
+++[29,31,40]\end{array}$ & $\begin{array}{l}=[41] \\
++[32,40]\end{array}$ & $++[40,41]$ \\
\hline Intestinal inflammation & & & & $++[34,38]$ \\
\hline
\end{tabular}

The association between levels of the biomarker and the severity of sickness behavior is $(+)$ significantly positive or (=) not significant. system detects endotoxins in the blood, the Toll-LikeReceptor-4 pathway is activated in monocytes and the sCD14/LBP complex is released into circulation [52]. sCD14 binds to LPS in the cell wall of gram-negative bacteria alongside its co-receptor LBP (Fig. 1) [53, 54], stimulating the release of pro-inflammatory cytokines [52]. Similarly, the adaptive immune response is triggered, resulting in increased release of antibodies against bacterial endotoxins in the bloodstream.

In experimental models of endotoxemia, the chronic proinflammatory status that follows bacterial translocation results in a whole-body response that manifest with sickness behavior in the attempt to conserve energy and recover. In line with these models and consistent with the literature on inflammation and psychiatric disorders, we found that increased levels of proxy biomarkers of gut dysbiosis were positively associated with severity of sickness behavior in severe mental illness and chronic fatigue.

Among these biomarkers, those related to the adaptive immune response to endotoxins were most consistently associated with sickness behavior across diagnoses (Table 3). Several epidemiological studies have shown an association between autoimmune diseases and severe mental illnesses $[3,55,56]$, and an autoimmune root has been suggested for CFS. Gut dysbiosis and structurally distinct endotoxins have been shown to trigger autoimmunity [57, 58], with downstream consequences of neuroinflammation [5, 59] and clinical phenotypes of sickness behavior.

Finally, we found evidence of increased intestinal inflammation in patients compared to controls. The acute phase protein A-1-AT was increased in patients, indicating greater inflammation and protein loss (Fig. 2f) [60, 61]. In contrast, the pooled estimate for I-FABP did not reveal a significant difference between patients and controls (Fig. 2g). I-FABP is uniquely localized in the gut and released with enterocyte damage, so we expected it to be elevated with inflammation of the gastrointestinal (GI) tract $[39,62]$. A-1-AT is released by different tissues in response to acute damage; one potential explanation for these findings is that A-1-AT may be elevated as a consequence of a systemic inflammation, rather than localized (GI) inflammation.
Medications showed no influence on the elevation of gut dysbiosis biomarkers (see Supplementary). This finding challenges reports that nonantibiotic drugs [36], including second generation antipsychotics [63] and SSRIs [64] alter the composition of the gut microbiome. It is possible that druginduced changes in gut microbiome composition are not sufficient to impact the pathophysiological path characterized by compromised intestinal barrier integrity, bacterial translocations, and activation of the systemic immune response.

Altogether, our findings suggest that gut dysbiosis occurs in severe mental illness and chronic fatigue and might underlie symptoms of sickness behaviors.

Some of the investigated markers, such as antibodies against endotoxins, were altered across all the included clinical conditions. This suggests that gut dysbiosis could represent a novel trans-diagnostic marker and a potential trans-therapeutic target for symptoms of sickness behavior. Future prospective studies should validate the transdiagnostic relevance of gut dysbiosis following the recently introduced TRANSD criteria (please see ref. [65]).

Future studies aimed at clarifying the trans-diagnostic relevance of gut dysbiosis for symptoms sickness behavior are of upmost importance as research based on traditional diagnostic categories failed to identify biological correlates and effective therapeutic targets for these highly invalidating symptoms.

\section{Limitations}

This systematic review and meta-analysis has limitations. First, we used proxy biomarkers of gut dysbiosis rather than direct measures of reduced microbial diversity. We used this approach for two reasons: (1) larger number of evidence and greater potential for replication of results obtained on blood-based biomarkers; (2) issues in gut microbiome datasets of individual data quality and inherent heterogeneity of individual datasets.

The use of proxy biomarkers comes with the limitation that some mediators, albeit commonly used in the scientific literature on the topic, might suffer from complex and hardly accountable confounding factors. This is the case of zonulin and sCD14, which alterations can be triggered by 
mechanisms other than gut dysbiosis. However, among the included biomarkers, LPS, LBP, and antibodies to endotoxin, which are more strictly related to gut dysbiosis, were consistently increased in patients vs. controls, therefore accounting for the "specificity" bias.

Second, it was possible to pool data for each included diagnostic categories only for $\mathrm{SCD} 14$ and antibodies again bacterial endotoxin. So, trans-diagnostic considerations extended to other biomarkers need to be further explored by future studies. Third, none of the studies provided direct measures of "severity of sickness behaviors." Studies investigating each diagnostic category used a different severity scale. This is particularly relevant for studies on schizophrenia, where only negative, but not depressive, symptoms were measured. However, as highlighted in Table 1, it is clear how symptoms definitions overlap across different scales and diagnoses.

Fourth, new evidence questioned the reliability of zonulin as a biomarker of intestinal permeability because of inadequacy of commercially available enzyme assays. However, our results rely on multiple biomarkers of gut dysbiosis, reducing the possibility of biased interpretations.

Finally, the pooled estimate for A-1-AT and SCD14 was characterised by high heterogeneity, even when accounted for by sensitivity analyses. These results warrant exploration in larger, longitudinal cohorts.

\section{Conclusions}

Our findings suggest that gut dysbiosis may underlie symptoms of sickness behavior across traditional diagnostic boundaries and provide evidence for future investigations on a trans-diagnostic target in an area of unmet clinical need.

\section{Compliance with ethical standards}

Conflict of interest The authors declare that they have no conflict of interest.

Publisher's note Springer Nature remains neutral with regard to jurisdictional claims in published maps and institutional affiliations.

Open Access This article is licensed under a Creative Commons Attribution 4.0 International License, which permits use, sharing, adaptation, distribution and reproduction in any medium or format, as long as you give appropriate credit to the original author(s) and the source, provide a link to the Creative Commons license, and indicate if changes were made. The images or other third party material in this article are included in the article's Creative Commons license, unless indicated otherwise in a credit line to the material. If material is not included in the article's Creative Commons license and your intended use is not permitted by statutory regulation or exceeds the permitted use, you will need to obtain permission directly from the copyright holder. To view a copy of this license, visit http://creativecommons. org/licenses/by/4.0/.

\section{References}

1. MacQueen G, Surette M, Moayyedi P. The gut microbiota and psychiatric illness. J Psychiatry Neurosci. 2017;42:75-7.

2. Clapp M, Aurora N, Herrera L, Bhatia M, Wilen E, Wakefield S. Gut microbiota's effect on mental health: the gut-brain axis. Clin Pract. 2017;7:987.

3. Jeppesen R, Benros ME. Autoimmune diseases and psychotic disorders. Front Psychiatry. 2019;10:131.

4. Carabotti M, Scirocco A, Maselli MA, Severi C. The gut-brain axis: interactions between enteric microbiota, central and enteric nervous systems. Ann Gastroenterol. 2015;28:203-9.

5. Alhasson F, Das S, Seth R, Dattaroy D, Chandrashekaran V, Ryan $\mathrm{CN}$, et al. Altered gut microbiome in a mouse model of Gulf War Illness causes neuroinflammation and intestinal injury via leaky gut and TLR4 activation. PloS ONE. 2017;12:e0172914.

6. Fasano A. Zonulin, regulation of tight junctions, and autoimmune diseases: Zonulin, regulation of tight junctions. Ann N. Y Acad Sci. 2012;1258:25-33.

7. Berg R. The indigenous gastrointestinal microflora. Trends Microbiol. 1996;4:430-5.

8. Maes M, Berk M, Goehler L, Song C, Anderson G, Gałecki P, et al. Depression and sickness behavior are Janus-faced responses to shared inflammatory pathways. BMC Med. 2012;10:66.

9. Jakobsson J, Bjerke M, Sahebi S, Isgren A, Johan Ekman C, Sellgren C, et al. Monocyte and microglial activation in patients with mood-stabilized bipolar disorder. J Psychiatry Neurosci. 2015;40:250-8.

10. Meiseberg J, Moritz S. Biases in diagnostic terminology: clinicians choose different symptom labels depending on whether the same case is framed as depression or schizophrenia. Schizophr Res. 2020;222:444-9.

11. Guessoum SB, Le Strat Y, Dubertret C, Mallet J. A transnosographic approach of negative symptoms pathophysiology in schizophrenia and depressive disorders. Prog Neuropsychopharmacol Biol Psychiatry. 2020;99:109862.

12. Farooq RK, Asghar K, Kanwal S, Zulqernain A. Role of inflammatory cytokines in depression: focus on interleukin-1 $\beta$. Biomed Rep. 2017;6:15-20.

13. Tanaka T, Matsuda T, Hayes LN, Yang S, Rodriguez K, Severance $\mathrm{EG}$, et al. Infection and inflammation in schizophrenia and bipolar disorder. Neurosci Res. 2017;115:59-63.

14. Goldsmith DR, Rapaport MH. Inflammation and negative symptoms of schizophrenia: implications for reward processing and motivational deficits. Front Psychiatry. 2020;11:46.

15. Montoya JG, Holmes TH, Anderson JN, Maecker HT, RosenbergHasson Y, Valencia IJ, et al. Cytokine signature associated with disease severity in chronic fatigue syndrome patients. Proc Natl Acad Sci. 2017;114:E7150-8.

16. Cross-Disorder Group of the Psychiatric Genomics Consortium. Identification of risk loci with shared effects on five major psychiatric disorders: a genome-wide analysis. Lancet Lond Engl. 2013;381:1371-9.

17. Insel T, Cuthbert B, Garvey M, Heinssen R, Pine DS, Quinn K, et al. Research Domain Criteria (RDoC): toward a new classification framework for research on mental disorders. Am J Psychiatry. 2010;167:748-51.

18. Wan X, Wang W, Liu J, Tong T. Estimating the sample mean and standard deviation from the sample size, median, range and/or interquartile range. BMC Med Res Methodol. 2014;14:135.

19. Wong CT, Tsoi WF, Saha N. Acute phase proteins in male Chinese schizophrenic patients in Singapore. Schizophr Res. 1996;22:165-71.

20. Maes M, Delange J, Ranjan R, Meltzer HY, Desnyder R, Cooremans $\mathrm{W}$, et al. Acute phase proteins in schizophrenia, mania and 
major depression: modulation by psychotropic drugs. Psychiatry Res. 1997;66:1-11.

21. Yang Y, Wan C, Li H, Zhu H, La Y, Xi Z, et al. Altered levels of acute phase proteins in the plasma of patients with schizophrenia. Anal Chem. 2006;78:3571-6.

22. Severance EG, Gressitt KL, Stallings CR, Origoni AE, Khushalani $\mathrm{S}$, Leweke FM, et al. Discordant patterns of bacterial translocation markers and implications for innate immune imbalances in schizophrenia. Schizophr Res. 2013;148:130-7.

23. Dickerson F, Stallings C, Origoni A, Schroeder J, Katsafanas E, Schweinfurth L, et al. Inflammatory markers in recent onset psychosis and chronic schizophrenia. Schizophr Bull. 2016;42: 134-41.

24. Dickerson F, Adamos M, Katsafanas E, Khushalani S, Origoni A, Savage $\mathrm{C}$, et al. The association between immune markers and recent suicide attempts in patients with serious mental illness: a pilot study. Psychiatry Res. 2017;255:8-12.

25. Weber NS, Gressitt KL, Cowan DN, Niebuhr DW, Yolken RH, Severance EG. Monocyte activation detected prior to a diagnosis of schizophrenia in the US Military New Onset Psychosis Project (MNOPP). Schizophr Res. 2018;197:465-9.

26. Delaney S, Fallon B, Alaedini A, Yolken R, Indart A, Feng T, et al. Inflammatory biomarkers in psychosis and clinical high risk populations. Schizophr Res. 2019;206:440-3.

27. Čiháková D, Eaton WW, Talor MV, Harkus UH, Demyanovich $\mathrm{H}$, Rodriguez $\mathrm{K}$, et al. Gut permeability and mimicry of the Glutamate Ionotropic Receptor NMDA type Subunit Associated with protein 1 (GRINA) as potential mechanisms related to a subgroup of people with schizophrenia with elevated antigliadin antibodies (AGA IgG). Schizophr Res. 2019;208:414-9.

28. Mørch RH, Dieset I, Færden A, Reponen EJ, Hope S, Hoseth EZ, et al. Inflammatory markers are altered in severe mental disorders independent of comorbid cardiometabolic disease risk factors. Psychol Med. 2019;49:1749-57.

29. Maes M, Kanchanatawan B, Sirivichayakul S, Carvalho AF. In schizophrenia, increased plasma $\operatorname{IgM} / \operatorname{IgA}$ responses to gut commensal bacteria are associated with negative symptoms, neurocognitive impairments, and the deficit phenotype. Neurotox Res. 2019;35:684-98.

30. Maes M, Sirivichayakul S, Kanchanatawan B, Vodjani A. Upregulation of the intestinal paracellular pathway with breakdown of tight and adherens junctions in deficit schizophrenia. Mol Neurobiol. 2019;56:7056-73.

31. Maes M, Sirivichayakul S, Kanchanatawan B, Vodjani A. Breakdown of the paracellular tight and adherens junctions in the gut and blood brain barrier and damage to the vascular barrier in patients with deficit schizophrenia. Neurotox Res. 2019;36:306-22.

32. Severance EG, Gressitt KL, Yang S, Stallings CR, Origoni AE, Vaughan $\mathrm{C}$, et al. Seroreactive marker for inflammatory bowel disease and associations with antibodies to dietary proteins in bipolar disorder. Bipolar Disord. 2014;16:230-40.

33. Kılıç F, Işık Ü, Demirdaş A, Doğuç DK, Bozkurt M. Serum zonulin and claudin-5 levels in patients with bipolar disorder. $\mathbf{J}$ Affect Disord. 2020;266:37-42.

34. Maes M. Higher $\alpha 1$-antitrypsin, haptoglobin, ceruloplasmin and lower retinol binding protein plasma levels during depression: further evidence for the existence of an inflammatory response during that illness. J Affect Disord. 1992;24:183-92.

35. Papakostas GI, Shelton RC, Kinrys G, Henry ME, Bakow BR, Lipkin SH, et al. Assessment of a multi-assay, serum-based biological diagnostic test for major depressive disorder: a Pilot and Replication Study. Mol Psychiatry. 2013;18:332-9.

36. Stevens BR, Goel R, Seungbum K, Richards EM, Holbert RC, Pepine CJ, et al. Increased human intestinal barrier permeability plasma biomarkers zonulin and FABP2 correlated with plasma
LPS and altered gut microbiome in anxiety or depression. Gut. 2018;67:1555-7.

37. Gomes C, Martinho FC, Barbosa DS, Antunes LS, Póvoa HCC, Baltus THL, et al. Increased root canal endotoxin levels are associated with chronic apical periodontitis, increased oxidative and nitrosative stress, major depression, severity of depression, and a lowered quality of life. Mol Neurobiol. 2018;55:2814-27.

38. Ohlsson L, Gustafsson A, Lavant E, Suneson K, Brundin L, Westrin $\AA$, et al. Leaky gut biomarkers in depression and suicidal behavior. Acta Psychiatr Scand. 2019;139:185-93.

39. Alvarez-Mon MA, Gómez AM, Orozco A, Lahera G, Sosa MD, Diaz D, et al. Abnormal distribution and function of circulating monocytes and enhanced bacterial translocation in major depressive disorder. Front Psychiatry. 2019;10:812.

40. Maes M, Simeonova D, Stoyanov D, Leunis J. Upregulation of the nitrosylome in bipolar disorder type 1 (BP1) and major depression, but not BP2: increased IgM antibodies to nitrosylated conjugates are associated with indicants of leaky gut. Nitric Oxide. 2019;91:67-76.

41. Simeonova D, Stoyanov D, Leunis J, Carvalho AF, Kubera M, Murdjeva M, et al. Increased serum immunoglobulin responses to gut commensal gram-negative bacteria in unipolar major depression and bipolar disorder type 1, especially when melancholia is present. Med Pharmacol. 2019.

42. Maes M, Mihaylova I, Leunis J-C. Increased serum IgA and IgM against LPS of enterobacteria in chronic fatigue syndrome (CFS): indication for the involvement of gram-negative enterobacteria in the etiology of CFS and for the presence of an increased gutintestinal permeability. J Affect Disord. 2007;99:237-40.

43. Maes M, Leunis J-C. Normalization of leaky gut in chronic fatigue syndrome (CFS) is accompanied by a clinical improvement: effects of age, duration of illness and the translocation of LPS from gram-negative bacteria. Neuro Endocrinol Lett. 2008;29: 902-10.

44. Maes M, Twisk FNM, Kubera M, Ringel K, Leunis J-C, Geffard M. Increased $\operatorname{IgA}$ responses to the LPS of commensal bacteria is associated with inflammation and activation of cell-mediated immunity in chronic fatigue syndrome. J Affect Disord. 2012; 136:909-17.

45. Maes M, Leunis J-C, Geffard M, Berk M. Evidence for the existence of Myalgic Encephalomyelitis/Chronic Fatigue Syndrome (ME/CFS) with and without abdominal discomfort (irritable bowel) syndrome. Neuro Endocrinol Lett. 2014;35: 445-53.

46. Giloteaux L, Goodrich JK, Walters WA, Levine SM, Ley RE, Hanson MR. Reduced diversity and altered composition of the gut microbiome in individuals with myalgic encephalomyelitis/ chronic fatigue syndrome. Microbiome. 2016;4:30.

47. Severance EG, Alaedini A, Yang S, Halling M, Gressitt KL, Stallings CR, et al. Gastrointestinal inflammation and associated immune activation in schizophrenia. Schizophr Res. 2012;138: 48-53.

48. Maes M, Kubera M, Leunis J-C, Berk M, Geffard M, Bosmans E. In depression, bacterial translocation may drive inflammatory responses, oxidative and nitrosative stress (O\&NS), and autoimmune responses directed against O\&NS-damaged neoepitopes. Acta Psychiatr Scand. 2013;127:344-54.

49. Song C, Dinan T, Leonard BE. Changes in immunoglobulin, complement and acute phase protein levels in the depressed patients and normal controls. J Affect Disord. 1994;30:283-8.

50. Gomes C, Martinho FC, Barbosa DS, Antunes LS, Póvoa HCC, Baltus THL, et al. Increased root canal endotoxin levels are associated with chronic apical periodontitis, increased oxidative and nitrosative stress, major depression, severity of depression, and a lowered quality of life. Mol Neurobiol. 2018;55:2814-27. 
51. Morris G, Fernandes BS, Puri BK, Walker AJ, Carvalho AF, Berk M. Leaky brain in neurological and psychiatric disorders: drivers and consequences. Aust N Z J Psychiatry. 2018;52:924-48.

52. Shive CL, Jiang W, Anthony DD, Lederman MM. Soluble CD14 is a nonspecific marker of monocyte activation. AIDS Lond Engl. 2015;29:1263-5.

53. Wright SD, Ramos RA, Tobias PS, Ulevitch RJ, Mathison JC. CD14, a receptor for complexes of lipopolysaccharide (LPS) and LPS binding protein. Science. 1990;249:1431-3.

54. Kitchens RL, Thompson PA. Modulatory effects of sCD14 and LBP on LPS-host cell interactions. J Endotoxin Res. 2005;11: 225-9.

55. Benros ME, Waltoft BL, Nordentoft M, Østergaard SD, Eaton WW, Krogh J, et al. Autoimmune diseases and severe infections as risk factors for mood disorders: a nationwide study. JAMA Psychiatry. 2013;70:812.

56. Lennox BR, Tomei G, Vincent S-A, Yeeles K, Pollard R, PalmerCooper E, et al. Study of immunotherapy in antibody positive psychosis: feasibility and acceptability (SINAPPS1). J Neurol Neurosurg Psychiatry. 2019;90:365-7.

57. Vatanen T, Kostic AD, d'Hennezel E, Siljander H, Franzosa EA, Yassour M, et al. Variation in microbiome LPS immunogenicity contributes to autoimmunity in humans. Cell. 2016;165:842-53.

58. Hoffmann C, Zong S, Mané-Damas M, Molenaar P, Losen M, Martinez-Martinez P, et al. Addendum: Hoffmann, C.; et al.
Autoantibodies in neuropsychiatric disorders. Antibodies 2016, 5, 9. Antibodies. 2018;7:33.

59. Dalmau J, Armangué T, Planagumà J, Radosevic M, Mannara F, Leypoldt F, et al. An update on anti-NMDA receptor encephalitis for neurologists and psychiatrists: mechanisms and models. Lancet Neurol. 2019;18:1045-57.

60. Karbach U, Ewe K, Bodenstein H. Alpha 1-antitrypsin, a reliable endogenous marker for intestinal protein loss and its application in patients with Crohn's disease. Gut. 1983;24:718-23.

61. Sharpstone D, Rowbottom A, Nelson M, Gazzard B. Faecal alpha 1 antitrypsin as a marker of gastrointestinal disease in HIV antibody positive individuals. Gut. 1996;38:206-10.

62. Kanda T, Fujii H, Fujita M, Sakai Y, Ono T, Hatakeyama K. Intestinal fatty acid binding protein is available for diagnosis of intestinal ischaemia: immunochemical analysis of two patients with ischaemic intestinal diseases. Gut. 1995;36:788-91.

63. Skonieczna-Żydecka K, Łoniewski I, Misera A, Stachowska E, Maciejewska D, Marlicz W, et al. Second-generation antipsychotics and metabolism alterations: a systematic review of the role of the gut microbiome. Psychopharmacology. 2019;236:1491-512.

64. Weersma RK, Zhernakova A, Fu J. Interaction between drugs and the gut microbiome. Gut. 2020;69:1510-9.

65. Fusar-Poli P, Solmi M, Brondino N, Davies C, Chae C, Politi P, et al. Transdiagnostic psychiatry: a systematic review. World Psychiatry. 2019; 18:192-207. 